\title{
Cultivation of Safety Consciousness in the Teaching of Chemistry
}

\author{
Yan Dong \& Leifang Liu \\ Dezhou University, Dezhou 253023, China \\ E-mail: sddzdy@163.com
}

Jianhua Yang

Dezhou No.1 Middle School, Dezhou 253023, China

E-mail: orgllf@163.com

\begin{abstract}
This paper sets forth the necessities of safety education, and talks about the factors that affecting the safety in chemistry laboratory. The methods for cultivating and strengthening safety consciousness in chemical experiments are given, which are efficient ways to decrease and avoid accidents in the chemistry laboratory.
\end{abstract}

Keywords: Chemical experiment, Safety, Factor, Method

It is well known that chemistry is a natural science that based on experiments. So it is an important approach to acquire chemical knowledge by experiments. Safety education, being an important part in the teaching of chemistry, has drawn more and more our attention. In the teaching of chemical experiment, teachers should not only just teach students how to carry out experiments and analyze the chemical phenomenon in order to cultivate their abilities to observe problems, analyze problems and solve problems, but also they should cultivate the safety consciousness of students. We must pay attention to the safety problems in chemical experiments no matter where the experiments are done, chemistry laboratory or in the classroom, in order to ensure the success of the operation and the safety of the students. It is very necessary to strength the cultivation of safety consciousness when the chemical experiment teaching is used to improve diatheses and abilities of the students.

\section{Importance of cultivating students' safety consciousness}

Experiment teaching is a very important part in chemistry teaching. The precondition to ensure the success of experiment teaching is the cultivation of safety consciousness of the students. Due to the intense contact of the students with the common glass apparatus, corrosive acids and bases that are often used in chemical experiments, there are many potential problems of safety in the experiment, which perhaps will bring on chemical accidents at different degrees. Once the accident happens, our normal experiment teaching will be confronted with serious negative effects. At present, the number of chemical accidents that happen in chemical laboratory in our country is too numerous to mention one by one. The main reasons are: (1) Safety education is inadequate for students by teachers. (2) Safety management and safety precautions are not enough during the course of the experiment. Some teachers think that it is easy to carry out the experiment by students themselves, so it is comparably safe for students, especially in the experimental class of inorganic chemistry, physical chemistry and analytical chemistry. (3) Safety consciousness of students is weak. In experimental class, students are asked to do experiments by themselves. So it is especially important for students to possess safety diatheses. It would have bad effect on the study of students in school without good diatheses of safety. What is more important is that it would cause personal injuries to students. In some schools the number of experiments that are carried out by the students themselves has decreased because of potential accidents in the experiment although it is well known to us that chemistry is a subject that based on experiments. Because the students have no enough chances to carry out experiments, so their thinking and the abilities to handle experiments are influenced to a great extent. Therefore the teaching effect is greatly affected by the decreases of the chemical experiments of the students. In fact, we all know that the chemical accidents mainly come from nonstandard operation of the students. As long as every student follows the correct operation methods strictly, accidents in the chemical experiment will be avoided. Teachers must ensure that the chemical teaching goes on wheels. In the chemical experiments teaching, teachers must pay attention to the safety of the chemical experiments, leave no stone unturned to cultivate the safety consciousness of the students, to avoid the occurrences of the chemical accidents. 


\section{Unsafe factors in chemical experiments}

\subsection{Unsafe chemical environmental factors}

Unsafe chemical environmental factors refer to the accidents caused by reagents, explosion of pyrotechnics, poisonous gases and so on. Several familiar unsafe chemical environmental factors are (1) Dangerous chemical reagents. (2) Hypertoxic reagents. Lots of chemicals that deposited in the laboratory and produced in the experiment are highly toxic. So it is very dangerous if these hypertoxic reagents are deposited or used in improper ways. Serious environmental pollution and personal injuries will happen. (3) Reagents that cause chemical pollution.

\subsection{Unsafe physical environmental factors}

Unsafe physical environmental factors are accidents that caused by mechanical equipments, pressure vessels, water source, power supply, heat source and radiation of light and so on. Several familiar unsafe physical environmental factors are (1) Instruments and equipments. Accidents caused by instruments and equipments include damages of the equipments itself and personal injuries by the equipments. These accidents are hidden troubles in chemistry laboratory mainly because of the shortage of protective apparatus, aging of equipments, and overloaded usage of equipments. (2) Fire caused by electric apparatus. Because of old apparatus of power supply, aging of lines in some schools, short circuit usually happens. So the ability to fireproof of these schools is very poor. Accidents, such as electric fires, electric shocks, burns and so on will happen as easy as pie if the operators use electricity incorrectly. (3) Vessels that contain compressed gases. (4) Flood caused by leakage of water.

\subsection{Unsafe behavior factors}

Unsafe behavior factors are mainly caused by human, such as carelessness in mind, lack of energy in life and shortage of necessary skills and knowledge in personal abilities. The reasons are (1) distemperedness in the system of safety management, (2) lack of safety consciousness.

\section{Cultivation of safety consciousness in the teaching of chemistry}

\subsection{Strengthening the education of safety to create safe culture atmosphere}

The education of safety is a precautionary task to prevent the occurrences of the accidents. Safety cultivation should be incorporated into the teaching work of schools. Strengthening the safety education and creating safe culture atmosphere should be turned into important parts of daily life for the teachers and students in the schools. Lectures on safety knowledge, knowledge competitions on safety, and analysis on safety accidents are efficient ways to cultivate the safety consciousness of teachers and students, and therefore accidents are avoided efficiently.

\subsection{Putting safety education into experiment operations of the students}

In chemical experiments, students will come into contact with poisonous gases, liquids, and solids directly. How to guide students to operate exactly, use the right dosage of reagents, improve the experimental methods, and design experimental apparatus becomes an important part in the teaching of chemistry, which is also an efficient way to strengthen the cultivation of safety consciousness by reminding you of the safety knowledge in your mind. Students not only get intuitable perceptual knowledge but also enhance their abilities to observe, describe, analyze and solve problems by experimental phenomenon that is difficult to express in words in chemical experiment. In order to call students' attention to self-protection consciousness and remind students that they should in no circumstances be reckless of dangerous poisonous chemicals, teachers should explain medically the dangers and toxic symptoms of poisonous chemicals in details in experimental classes. At the same time, to build up students' consciousness of self-protection and environmental protection as masters, teachers should emphasize that dangerous and poisonous chemicals must not be placed everywhere and each students must learn to deal with such chemicals correctly.

\subsection{Consideration in ensuring the safety in the design of the experiments}

Chemical experiment, characterized by its unique properties of vitality, intuition, science and practice, is an important approach for cultivating the innovation ability of students. Therefore teachers should conduct students to improve the method of experiment audaciously. By designing new and safe experiment by students, not only the consciousness of innovation is inspired, but also the importance of safety is realized. Using interest investigatory experiment as a chance, teachers should strengthen the education of safety of students by starting with the choose of the topic of the experiment, thereby students are able to be conscious of the aim to design experiment. So safety consideration in the design of experiments becomes the embodiment to cultivate students' consciousness of safety. 


\subsection{Cultivating students'ability to operate experiments safely by normalizing the operation of students}

Teachers should have their eyes glued to three thoughts that emerged during the course of experiment of students. (1) Considering chemical experiments as fun things, not as religious scientific researches, some students think that it is an easy thing to do chemical experiment. (2) Lots of students think that anticipated phenomena will appear without question even though incorrect operations are taken. They think that the chemical experiments that usually done by them, not the final experiment examination, are nonsignificant. (3) Some students think that it is dangerous to do chemical experiment. They act as spectators, sitting there, without any operations. Therefore, there are potential accidents because of the poor ability of students to carry out experiments correctly and safely and lack of safety consciousness. In chemistry laboratory, you will see many incorrect operations, such as alcohol burner is puffed by mouth after use, reactants overflow during the reaction because of the over usage of the reagents, reagents don't be put where they should be in time after use, matches are thrown away everywhere, and so on. It is obvious that normalizing experimental operation of students and strengthening the safety education of students are very important in chemical experiments. Before experiment, teachers should make students clear the properties of the reagents and the potential problems during the course of experiment, and let students understand the potential dangers in the experiment. In order to impress on students the importance of safety, teachers may use computer to simulate some incorrect operations and relate accidents that caused by the incorrect operations. Students will see clearly the danger caused by the incorrect operations and pay much attention to the safety problems in chemistry laboratory. However after inverses education, it is very necessary for teachers to make students clear the remedial measures after the incorrect operation. At the same time, teachers should ask students to do the experiment by themselves, and then students would realize and understand initiatively the safety problem in the experiment. Not only aftereffects caused by safety problem are realized by students, but also the method to prevent and deal with the safety problems are known to students. We believe that students will fall into the habit of precise experimental attitude and normal experimental operation.

\subsection{Exhibiting consciousness of safety using investigatory topics as chances}

It is well known that safety knowledge is included during the course of resolving chemical exercises. If teachers have a mind to guide students in time to let them know about the safety knowledge during the answering questions, safety consciousness of students will greatly improved. Considering potential security problems in high temperature and high-pressure environment, we can design some topics to explore. For example, teachers can suggest students to do some investigation on safety problems and organize students to go on a field investigation in chemical plants, to let students realize and understand the importance of safety. I believe that they will know how to protect themselves and others in such high temperature and high-pressure environment.

Recently, chemical experiment is developed more and more in the direction of embodying the breath of life, embracing energy sources, technology and protection of environment and so on. Materially these directions are all involved with safety problems. In order to letting students grow up safely and healthily, chemistry educators should cultivate actively the safety consciousness of students at any time you could.

\section{Acknowledgements}

We thank the Research Project of Teaching Reform in High Education Institutions of Shandong Province (No. 2009079) for providing financial support.

\section{References}

Qi, Q., Luo, Z. K., Lv, W. Z., \& Fang, W. (2010). Investigation on University Safety Management of Chemistry Laboratory in Domestic and Abroad. Guangdong Chemical Industry, 38(5), 272-274.

Yuan, N. X., \& Xie, D. P. (2009). Prevention and Measures to Chemical Experiments' Unexpected Safety Accidents. Laboratory Science, 2(2), 164-166.

Zeng, M. H., Hong, X. L., Wu, D. C., Peng, C. H., \& Zhao, X. G. (2009). By Sino-US Comparasion on the Experimental Safety Regulation to Reflect on the College Chemical Laboratory Safety Administration in Our Country. Research and Exploration in Laboratory, 28(6), 310-313.

Zhang, S. X. (2008). The Management and Safety Measures of Dangerous Chemicals in Chemical Laboratory. Journal of Chongqing University of Science and Technology (Natural Sciences Edition), 10(4), 76-78.

Zhou, J. X. (2006). Safety Prevention and First Aid in the Chemical Laboratory. Safety, Health \& Environment, $6(4), 35-37$. 